\title{
Relações empíricas entre características dendrométricas da Caatinga brasileira e dados TM Landsat 5
}

\author{
André Quintão de Almeida(1), Anabel Aparecida de Mello(1), Antônio Luiz Dória Neto(1) \\ e Raphael Cavalcanti Ferraz ${ }^{(1)}$
}

\begin{abstract}
(1)Universidade Federal de Sergipe, Cidade Universitária Prof. José Aloísio de Campos, Avenida Marechal Rondon, s/noo, Jardim Roza Elze, CEP 49100-000 São Cristóvão, SE, Brasil. E-mail: andre.almeida@ufs.br, anabel_mello@yahoo.com.br, sgtdoria@ig.com.br, rapha_amadeus@hotmail.com
\end{abstract}

Resumo - O objetivo deste trabalho foi ajustar modelos para estimar características dendrométricas da Caatinga brasileira a partir de dados do sensor TM do Landsat 5. Medidas de diâmetro e altura das árvores foram obtidas de 60 parcelas de inventário $\left(400 \mathrm{~m}^{2}\right)$, em dois municípios do Estado de Sergipe. A área basal e o volume de madeira foram estimados com uso de equação alométrica e de fator de forma ( $\mathrm{f}=0,9)$. As variáveis explicativas foram obtidas do sensor TM, após correção radiométrica e geométrica, tendo-se considerado, na análise, seis bandas espectrais, com resolução espacial de $30 \mathrm{~m}$, além dos índices de razão simples (SR), de vegetação por diferença normalizada (NDVI) e de vegetação ajustado ao solo (Savi). Na escolha das melhores variáveis explicativas, foram considerados coeficiente de determinação $\left(\mathrm{R}^{2}\right)$, raiz do erro quadrático médio (RMSE) e critério bayesiano de informação (CBI). A área basal por hectare não apresentou correlação significativa com nenhuma das variáveis explicativas utilizadas. Os melhores modelos foram ajustados à altura média das árvores por parcela $\left(\mathrm{R}^{2}=0,4 ; \mathrm{RMSE}=13 \%\right)$ e ao volume de madeira por hectare $\left(\mathrm{R}^{2}=0,6 ; \mathrm{RMSE}=42 \%\right)$. As métricas derivadas do sensor TM do Landsat 5 têm grande potencial para explicar variações de altura média das árvores e do volume de madeira por hectare, em remanescentes de Caatinga situados no Nordeste brasileiro.

Termos para indexação: índice de vegetação, NDVI, REDD, redução de emissões, Savi, sensoriamento remoto.

\section{Empiric relations between dendrometric characteristics of the Brazilian dry forest and Landsat 5 TM data}

\begin{abstract}
The objective of this work was to adjust models to estimate dendrometric characteristics of the Brazilian dry tropical forest (Caatinga) from Landsat $5 \mathrm{TM}$ sensor data. Measures for tree diameter and height were taken in 60 inventory plots $\left(400 \mathrm{~m}^{2}\right)$, in two municipalities of the state of Sergipe, Brazil. Basal area and wood volume were estimated using an allometric equation and form factor $(\mathrm{f}=0.9)$. Explanatory variables were taken from the TM sensor, after radiometric and geometric correction, having considered, in the analysis, six spectral bands, with $30 \mathrm{~m}$ spatial resolution, besides the indexes of simple ratio (SR), of normalized difference vegetation (NDVI), and of soil-adjusted vegetation (Savi). To choose the best explanatory variables, the coefficient of determination $\left(\mathrm{R}^{2}\right)$, the root mean square error (RMSE), and the Bayesian information criterion (BIC) were considered. The basal area per hectare did not show a significant correlation with any of the explanatory variables used. The best models were adjusted to tree mean height per plot $\left(\mathrm{R}^{2}=0.4 ; \mathrm{RMSE}=13 \%\right)$ and to wood volume per hectare $\left(\mathrm{R}^{2}=0.6\right.$; RMSE $\left.=42 \%\right)$. The metrics derived from the Landsat $5 \mathrm{TM}$ sensor have great potential to explain variation in the mean height of trees and in the wood volume per hectare, in remaining areas of the tropical dry forest in the Brazilian Northeast.
\end{abstract}

Index terms: vegetation index, NDVI, REDD, reducing emissions, Savi, remote sensing.

\section{Introdução}

A Caatinga é um bioma exclusivamente brasileiro, que ocupa $9,92 \%$ do território nacional e cobre uma área de aproximadamente $844.453 \mathrm{~km}^{2}$ (Florestas do Brasil em resumo, 2013). Originalmente, o Bioma Caatinga recobria quase todo o Semiárido nordestino (Instituto Brasileiro de Geografia e
Estatística, 2010), o que evidencia sua importância como regulador hidroclimático, na região semiárida do Brasil. A vegetação de Caatinga é caracterizada pela predominância de árvores e arbustos baixos, ramificados, espinhosos e com folhagem decídua na estação seca (Juncá et al., 2005). Dos grandes domínios florísticos-vegetacionais brasileiros, a Caatinga é um dos mais desconhecidos, com variações fisionômicas

Pesq. agropec. bras., Brasília, v.49, n.4, p.306-315, abr. 2014

DOI: $10.1590 / \mathrm{S} 0100-204 X 2014000400009$ 
e florísticas resultantes da evolução e da adaptação aos fatores climáticos da região, em especial aos baixos valores de precipitação (menos de $700 \mathrm{~mm}$ por ano).

Apesar de o princípio da redução de emissões por desmatamento e degradação (REDD) ser um tema atual e amplamente discutido na convenção-quadro da Organização das Nações Unidas sobre mudança do clima, a vegetação de Caatinga vem passando por um contínuo e longo processo de redução em sua cobertura (Foley et al., 2005; Sánchez-Azofeifa et al., 2005). Atualmente, menos de $40 \%$ de sua área original ainda está coberta por vegetação nativa.

$\mathrm{Na}$ Conferência das Partes sobre o Clima (COP 15), realizada em Copenhagen, foram identificadas quatro principais ferramentas relacionadas à REDD: estimativa da biomassa e do carbono das florestas; gestão de grandes quantidades de dados espaciais; monitoramento do desmatamento e da degradação florestal; e modelagem do desmatamento futuro (Hajek et al., 2011). Essas ferramentas podem ser direta ou indiretamente relacionadas a técnicas de sensoriamento remoto, fundamentais para o monitoramento dos remanescentes florestais naturais pelo mundo.

Estudos realizados em florestas tropicais úmidas mostram o potencial do sensoriamento remoto em estimar características da vegetação (Bannari et al., 1995; Rosenqvist et al., 2003; Palacios-Orueta et al., 2005; Lu, 2006; Powell et al., 2010; Du et al., 2010). Recentemente, bons resultados têm sido obtidos em florestas características de clima seco, principalmente nas localizadas na América Central (Arroyo-Mora et al., 2005; Feeley et al., 2005; Gillespie, 2005; Kalacska et al., 2005). Entretanto, estudos que utilizam técnicas de sensoriamento remoto para a vegetação de Caatinga são escassos. Entre estes, destaca-se o trabalho de Accioly et al. (2002) na Floresta Nacional (Flona) do Araripe, localizada no Estado do Ceará. Os autores, apesar de também terem considerado outras formações florestais na análise, como a mata úmida, demonstraram o potencial das técnicas de sensoriamento remoto em estimar a altura e a área basal da vegetação analisada. Contudo, ainda há a necessidade de estudos que investiguem possíveis correlações entre outras características da vegetação de Caatinga, como volume e biomassa, com dados de sensoriamento remoto.
O objetivo deste trabalho foi ajustar modelos para estimar características dendrométricas da Caatinga brasileira a partir de dados do sensor TM do Landsat 5 .

\section{Material e Métodos}

O trabalho foi desenvolvido em dois remanescentes de Caatinga localizados no Estado de Sergipe, nos municípios de Porto da Folha e de Canindé de São Francisco (Figura 1). Os fragmentos de vegetação estão localizados na zona fisiográfica do Sertão Sergipano, caracterizada como área de Caatinga hiperxerófila densa (Ferraz et al., 2013). O fragmento de Porto da Folha $\left(09^{\circ} 38^{\prime} \mathrm{S}, 37^{\circ} 43^{\prime \prime} \mathrm{W}\right.$, a $250 \mathrm{~m}$ de altitude) tem área de aproximadamente 109 ha. O fragmento de Canindé de São Francisco (09³9'S, 3704"W, a 150 m de altitude) apresenta área de aproximadamente 191 ha. O solo predominante em ambas as áreas é classificado como Litossolo, raso e com textura muito arenosa. O clima da região é caracterizado por precipitações irregulares, que variam entre 300 e $700 \mathrm{~mm}$, mal distribuídas anualmente. A média histórica de precipitação é inferior a $500 \mathrm{~mm}$ anuais. $\mathrm{O}$ período seco da região supera oito meses, e as chuvas ocorrem durante o período de março a julho, com maiores índices de precipitação em abril. A temperatura média anual é de $25,6^{\circ} \mathrm{C}$, e não há grandes variações no decorrer dos meses.

As informações dendrométricas da vegetação foram obtidas de levantamento pelo método de parcela fixa, com parcelas quadradas de $400 \mathrm{~m}^{2}$ (20x20 m), distribuídas sistematicamente na área, com intervalo de 190 m, em Porto da Folha, e 250 m em Canindé de São Francisco. Ao todo, foram inventariadas 60 parcelas, 30 em cada município. As campanhas de campo foram realizadas em junho (Porto da Folha) e julho (Canindé de São Francisco), em 2008. O diâmetro à altura do peito (DAP) foi obtido com fita métrica, e as alturas totais, com auxílio de vara telescópica. Foram estimados os valores médios dessas variáveis em cada parcela. Todos os indivíduos com DAP maior que $1,9 \mathrm{~cm}$ - recomendado pela Rede de Manejo Florestal da Caatinga (2005) - e base do tronco dentro da parcela foram considerados mensuráveis.

Os valores de volume (VOL) foram estimados a partir da área basal $(\mathrm{AB})$, da altura $(\mathrm{H})$ e do fator de forma $(f=0,9)$ recomendado pela Rede de Manejo Florestal da Caatinga (2005), com uso da equação $\mathrm{VOL}=\mathrm{AB} \times \mathrm{H} \times \mathrm{f}$. 
As variáveis explicativas foram extraídas das bandas (B) espectrais de uma mesma cena do sensor Thematic Mapper (TM), à bordo do satélite Landsat 5, com resolução espacial de $30 \mathrm{~m}$. Registradas em 23/9/2008, as bandas apresentavam ângulo de elevação do sol de $58,73^{\circ}$, azimute de $73,17^{\circ}$, e ponto e órbita de passagem números 67 e 215, respectivamente. Nos meses das campanhas de campo (junho e julho de 2008), imagens com baixa cobertura de nuvens não foram registradas pelo sensor TM, o que inviabilizou seu uso. Apesar da defasagem de aproximadamente três meses entre a primeira campanha de inventário (junho) e a coleta da imagem Landsat 5 (setembro), este período não é suficiente para provocar, na vegetação de Caatinga, incrementos significativos nos valores de DAP e de altura das árvores.

$\mathrm{Na}$ fase de pré-processamento, as bandas espectrais foram georreferenciadas por meio de interpolador de primeiro grau de alocação de vizinho mais próximo. Durante as campanhas de inventário, foram coletados, no campo, 20 pontos de controle. A projeção final foi UTM/WGS84, zona 24 Sul. O erro quadrático médio encontrado foi inferior a $2 \mathrm{~m}$. Para cada banda espectral, foi realizada a correção atmosférica, em que os níveis digitais foram convertidos em radiância espectral, a partir do modelo 6S (Vermote et al., 1997), e, posteriormente, convertidos em reflectância $(\rho)$. Os parâmetros de entrada no modelo $6 \mathrm{~S}$ foram: modelo de atmosfera tropical, modelo continental de aerossóis e visibilidade horizontal de $25 \mathrm{~km}$.

Foram utilizadas seis bandas espectrais do sensor TM: B1, 0,45 a 0,52 $\mu \mathrm{m}$; B2, 0,52 a $0,60 \mu \mathrm{m}$; B3, 0,63 a

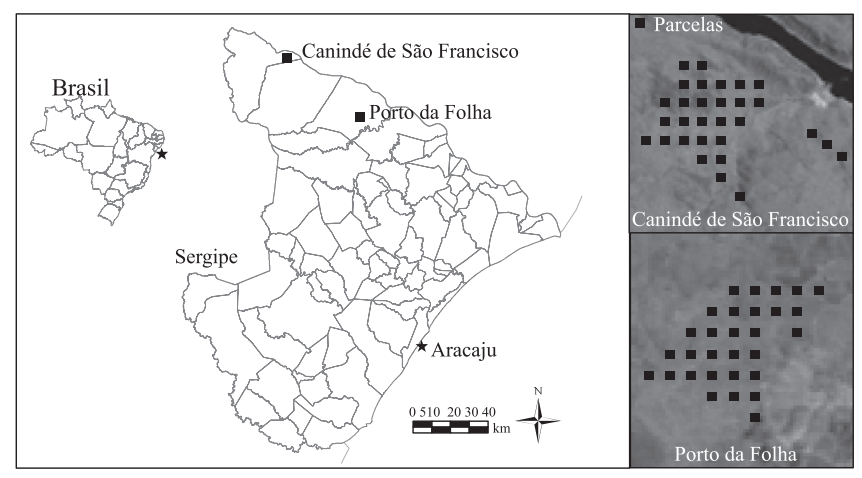

Figura 1. Localização das áreas de estudo e croqui das parcelas de inventário dos fragmentos de Canindé de São Francisco e Porto da Folha, no Estado de Sergipe.
$0,69 \mu \mathrm{m} ; \mathrm{B} 4,0,76$ a $0,90 \mu \mathrm{m} ; \mathrm{B} 5,1,55$ a $1,75 \mu \mathrm{m}$; e B7, 2,08 a 2,35 $\mu \mathrm{m}$. A partir destas bandas, foram estimados três índices de vegetação: o de razão simples (SR); o de vegetação por diferença normalizada (NDVI); e o de vegetação ajustado ao solo (Savi). O SR é um índice simples, porém exibe boa relação com características das florestas (Turner et al., 1999). É calculado pela equação: $\mathrm{SR}=(\mathrm{B} 4 / \mathrm{B} 3)$. O NDVI apresenta melhor correspondência com as características da vegetação (Tucker, 1979) e é estimado pela equação: NDVI $=($ B4 - B3)/(B4 + B3). O Savi, proposto por Huete (1988), minimiza a interferência do piso florestal, o que melhora sua relação com as métricas da vegetação. É calculado por: Savi $=(1+\mathrm{L}) \times(\mathrm{B} 4$ - B3)/(B4 + B3 + L), em que $\mathrm{L}=0,5$ para condições de vegetação aberta (Huete \& Warrick, 1990).

Foram avaliadas duas opções de tamanho de janela espectral na extração das variáveis explicativas do sensor TM: apenas o pixel central da imagem considerado; e a média de $3 \times 3$ pixels da banda espectral ou do índice correspondente. Avaliou-se, então, a opção que melhor correlacionou-se com os dados medidos no campo.

Os valores das bandas espectrais e dos índices de vegetação foram associados às características médias das 60 parcelas de inventário. A associação foi feita com as coordenadas (centro da parcela) coletadas em campo, com GPS de acurácia $\sim 5 \mathrm{~m}$, modelo Garmin 12XL, (Garmin International Inc., Olathe, KS, EUA), e os respectivos valores de coordenadas na imagem.

Os modelos de estimativas foram ajustados a partir da análise de correlação entre as variáveis medidas da floresta em campo (altura média, área basal e volume por hectare de cada parcela) e os valores das bandas espectrais e dos índices de vegetação. Foram utilizados modelos de regressão do tipo linear múltiplo. Depois do ajuste dos possíveis modelos, foram selecionados os que apresentaram o menor critério bayesiano de informação. Posteriormente, os modelos foram avaliados com uso do teste $\mathrm{t}$ de Student, a $5 \%$ de probabilidade. Procedeu-se à análise dos resíduos para avaliar a existência de tendência no erro. A correlação entre variáveis explicativas (multicolinearidade) foi avaliada pelo teste com o fator de inflação da variância ("variance inflation fator", VIF).

O poder de predição dos modelos ajustados foi avaliado a partir da técnica de validação cruzada, com base no coeficiente de determinação $\left(\mathrm{R}^{2}\right)$ e na raiz do

Pesq. agropec. bras., Brasília, v.49, n.4, p.306-315, abr. 2014

DOI: 10.1590/S0100-204X2014000400009 
erro quadrático médio (RMSE). Do conjunto de 60 observações, $25 \%$ foram utilizadas para a validação cruzada dos dados.

\section{Resultados e Discussão}

No fragmento de Porto da Folha, havia maior número médio de árvores e de fustes por parcela, o que o caracterizou como área mais densamente povoada que o fragmento de Canindé de São Francisco (Tabela 1). Em Porto da Folha, também foram encontrados os maiores valores médios de área basal por hectare e de altura média por parcela. Os baixos valores de desvio-padrão obtidos nesse fragmento indicam, ainda, que o remanescente é mais homogêneo.

Os coeficientes de correlação para a janela de $3 \times 3$ pixels foram ligeiramente superiores aos valores obtidos de apenas um pixel (Tabela 2). Desse modo, as informações espectrais consideradas na análise foram as extraídas da janela de 3x3 pixels. Ao se escolhê-la, são consideradas informações adicionais dos oito vizinhos mais próximos de cada pixel central da parcela de inventário.
A área basal não se correlacionou significativamente com nenhuma das variáveis explicativas obtidas do sensor TM. Já a altura total média das árvores por parcela e o volume de madeira por hectare correlacionaram-se fortemente com essas variáveis, e o volume de madeira foi a variável dendrométrica com os maiores coeficientes de correlação. Feeley et al. (2005) obtiveram resultados semelhantes, na investigação de relações entre medidas de estrutura e composição da floresta seca da Venezuela e três índices de vegetação derivados do Landsat. Os autores sugerem que o NDVI pode ser um bom indicador dos atributos de florestas de terras secas.

A ausência de correlação entre área basal e as variáveis explicativas pode estar relacionada à estrutura horizontal da vegetação de Caatinga, caracterizada por fustes entrelaçados e retorcidos (Juncá et al., 2005), o que dificultaria a obtenção de uma relação direta entre os valores de área basal e as variáveis explicativas do sensor orbital.

Para as seis bandas espectrais analisadas, a correlação com as variáveis dendrométricas foi sempre negativa, diferentemente do comportamento apresentado pelos

Tabela 1. Características dendrométricas inventariadas em 60 parcelas, nos fragmentos de Porto da Folha e Canindé de São Francisco, no Estado de Sergipe.

\begin{tabular}{|c|c|c|c|c|c|c|c|c|c|c|}
\hline \multirow[t]{2}{*}{ Estatística } & \multicolumn{5}{|c|}{ Porto da Folha } & \multicolumn{5}{|c|}{ Canindé de São Francisco } \\
\hline & Árvores & Fustes & $\begin{array}{c}\text { Área basal } \\
\left(\mathrm{m}^{2} \mathrm{ha}^{-1}\right)\end{array}$ & $\begin{array}{c}\text { Altura } \\
(\mathrm{m})\end{array}$ & $\begin{array}{l}\text { Volume } \\
\left(\mathrm{m}^{3} \mathrm{ha}^{-1}\right)\end{array}$ & Árvores & Fustes & $\begin{array}{c}\text { Área basal } \\
\left(\mathrm{m}^{2} \mathrm{ha}^{-1}\right)\end{array}$ & $\begin{array}{c}\text { Altura } \\
(\mathrm{m})\end{array}$ & $\begin{array}{l}\text { Volume } \\
\left(\mathrm{m}^{3} \mathrm{ha}^{-1}\right)\end{array}$ \\
\hline Média & 128,0 & 289,0 & 14,9 & 5,7 & 116,3 & 66,0 & 172,0 & 13,7 & 4,5 & 34,1 \\
\hline Máximo & 224,0 & 553,0 & 26,0 & 6,8 & 179,0 & 112,0 & 299,0 & 51,5 & 5,9 & 63,3 \\
\hline Mínimo & 74,0 & 181,0 & 6,7 & 4,2 & 24,8 & 35,0 & 74,0 & 4,5 & 3,0 & 6,7 \\
\hline Desvio-padrão & 17,0 & 18,1 & 4,3 & 13,4 & 45,0 & 32,9 & 30,5 & 8,3 & 17,0 & 15,3 \\
\hline
\end{tabular}

Tabela 2. Coeficiente de correlação linear entre variáveis dendrométricas - área basal por hectare, altura média por parcela e volume de madeira por hectare - e explicativas do sensor TM do Landsat 5 - bandas espectrais e índices de vegetação -, tendo-se considerado apenas um pixel da imagem ou o valor médio de uma janela de $3 \times 3$ pixels.

\begin{tabular}{|c|c|c|c|c|c|c|c|c|c|}
\hline \multirow[t]{2}{*}{ Dendrométricas } & \multicolumn{9}{|c|}{ Explicativas do sensor TM do Landsat 5} \\
\hline & $\mathrm{B} 1$ & $\mathrm{~B} 2$ & B3 & B4 & B5 & B7 & SR & NDVI & Savi \\
\hline & \multicolumn{9}{|c|}{1 pixel } \\
\hline Área basal $\left(\mathrm{m}^{2} \mathrm{ha}^{-1}\right)$ & $-0,08$ & $-0,10$ & $-0,17$ & $-0,24$ & $-0,18$ & $-0,15$ & 0,07 & $-0,06$ & $-0,01$ \\
\hline Altura (m) & $-0,57 *$ & $-0,53^{*}$ & $-0,58 *$ & $-0,27$ & $-0,49 *$ & $-0,54^{*}$ & $0,53 *$ & $0,53 *$ & $0,40^{*}$ \\
\hline \multirow[t]{2}{*}{ Volume $\left(\mathrm{m}^{3} \mathrm{ha}^{-1}\right)$} & $-0,66^{*}$ & $-0,67 *$ & $-0,69^{*}$ & $-0,35$ & $-0,60 *$ & $-0,64 *$ & $0,63 *$ & $0,63 *$ & $0,46^{*}$ \\
\hline & \multicolumn{9}{|c|}{$3 \times 3$ pixels } \\
\hline Área basal $\left(\mathrm{m}^{2} \mathrm{ha}^{-1}\right)$ & $-0,09$ & $-0,056$ & $-0,11$ & 0,047 & $-0,094$ & $-0,069$ & 0,16 & 0,16 & 0,16 \\
\hline Altura (m) & $-0,60 *$ & $-0,53^{*}$ & $-0,56^{*}$ & $-0,122$ & $-0,51^{*}$ & $-0,54^{*}$ & $0,59 *$ & $0,59 *$ & $0,52 *$ \\
\hline Volume $\left(\mathrm{m}^{3} \mathrm{ha}^{-1}\right)$ & $-0,74^{*}$ & $-0,70 *$ & $-0,72 *$ & $-0,238$ & $-0,67^{*}$ & $-0,71 *$ & $0,72 *$ & $0,72 *$ & $0,61 *$ \\
\hline
\end{tabular}

*Significativo a 5\% de probabilidade. B1 a B7, bandas espectrais; SR, índice de vegetação de razão simples; NDVI, índice de vegetação por diferença normalizada; Savi, índice de vegetação ajustado ao solo. 
índices de vegetação, em que a correlação foi positiva. Esse resultado é similar ao encontrado por Gasparri et al. (2010), no estudo da análise multitemporal entre dados Landsat e características da vegetação subtropical seca da Argentina. Apenas a banda espectral do infravermelho próximo (B4, 0,76 a $0,90 \mu \mathrm{m}$ ) não apresentou correlação significativa com as variáveis dendrométricas. Em dosséis florestais, a reflectância nessa região do espectro é praticamente constante. Além disso, fatores externos à folha, como disponibilidade de água, tipo de solo e tecido vegetal não fotossintético, podem promover alterações na reflectância.

Os modelos ajustados apresentaram bom desempenho, com baixos valores de erro quadrático médio da raiz e do critério bayesiano de informação (Tabela 3). Para a altura média $(\mathrm{H})$ das árvores por parcela, o modelo ajustado foi: $H(\mathrm{~m})=-15,60+$ $(131,34$ x B3) + (69,61 x NDVI) - (86,60 x Savi). Para o volume de madeira (VOL), a equação de estimativa obtida foi: $\operatorname{VOL}\left(\mathrm{m}^{3} \mathrm{ha}^{-1}\right)=-1.273,5+(8.644,7 \times \mathrm{B} 3)+$ (4.785,7 x NDVI) - (6.142,6 x Savi). A análise residual dos modelos ajustados sugere um pequeno desvio de normalidade (Figura 2).

$\mathrm{O}$ teste VIF evidenciou a ocorrência de multicolinearidade entre as variáveis explicativas (VIF $>10)$. Essamulticolinearidadepodeinflara variância dos parâmetros, o que dificultaria sua interpretação individual, assim como a de suas significâncias. Porém, como o propósito dos modelos aqui utilizados foi a predição, essa inflação teve pouca influência na análise, pois o método dos mínimos quadrados gera estimativas de parâmetros não tendenciosas.

Os modelos ajustados conseguiram explicar cerca de $40 \%$ da variação em altura e $60 \%$ da variação em volume de madeira. Esses valores foram superiores aos obtidos por Accioly et al. (2002), em vegetação com a mesma tipologia (36\%). O erro (RMSE) observado no modelo ajustado para a altura das árvores foi de apenas $0,7 \mathrm{~m}$, valor considerado baixo. O RMSE ajustado no modelo de volume foi de $32 \mathrm{~m}^{3} \mathrm{ha}^{-1}$. Apesar do bom desempenho

Tabela 3. Estatísticas dos modelos ajustados para altura e volume de madeira, no Bioma Caatinga ${ }^{(1)}$.

\begin{tabular}{lccccc}
\hline Modelo & $\mathrm{n}$ & $\mathrm{R}^{2}$ & $\mathrm{CBI}$ & RMSE & RMSE (\%) \\
\hline Altura $(\mathrm{m})$ & 60 & 0,4 & -14 & 0,7 & 13 \\
Volume $\left(\mathrm{m}^{3} \mathrm{ha}^{-1}\right)$ & 60 & 0,6 & -43 & 32,0 & 42 \\
\hline
\end{tabular}

${ }^{(1)}$, número de observações; $\mathrm{R}^{2}$, coeficiente de determinação; CBI, critério bayesiano de informação; RMSE, raiz do erro quadrático médio absoluto; RMSE (\%), raiz do erro quadrático médio em termos da média observada. do modelo de estimativa do volume, o valor percentual do RMSE encontrado (40\%) foi superior ao aceitável $(\sim 20 \%)$, para inventários florestais voltados à estimativa do volume de madeira em áreas de vegetação nativa (Instituto Brasileiro do Meio Ambiente e dos Recursos Naturais Renováveis, 2001). No entanto, a ferramenta apresentada pode estimar as características da vegetação de forma rápida, contínua e com baixo custo, o que resultaria em vantagens em relação aos erros embutidos no processo de inventário convencional.

Nos melhores modelos ajustados, foram utilizadas apenas três variáveis explicativas: a terceira banda do espectro do visível e os índices NDVI e Savi. Accioly et al. (2002) ajustaram o modelo $\left(\mathrm{R}^{2}=0,36\right)$ entre as alturas e o índice de razão simples, para a vegetação de Caatinga da Flona do Araripe, no Estado do Ceará. Desse modo, os autores utilizaram apenas os índices de vegetação como variáveis explicativas. Já Gasparri et al. (2010) utilizaram bandas espectrais e índices de vegetação derivados do Landsat 7 ETM para o ajuste do modelo de estimativa de biomassa da floresta tropical seca da Argentina, e, semelhantemente ao observado no presente trabalho, relataram que o NDVI foi o índice que melhor explicou a variação da biomassa.

Nenhum dos modelos ajustados utilizou o SR, apesar das correlações significativas observadas (Tabela 1). Vários estudos mostram boa correlação entre os parâmetros da vegetação e o NDVI. Em estudo com as principais formações florestais existentes na região Nordeste e Norte do Brasil, Formigoni et al. (2011) encontraram boa correlação entre a variação sazonal da vegetação de Caatinga e o NDVI de imagens de "moderate resolution imaging spectrometer" (Modis). De maneira geral, o NDVI é mais sensível à presença de clorofila e outros pigmentos responsáveis pela absorção da radiação solar, na banda do vermelho (Gao et al., 2000; Huete et al., 2002). Cabe ressaltar, contudo, que os modelos ajustados, no presente trabalho, representam apenas uma opção adicional para se estimar algumas variáveis da vegetação de terra seca. Entretanto, ao se considerar os baixos erros encontrados, a praticidade do método e a possibilidade de abrangência de $100 \%$ da área inventariada, os modelos ajustados podem ser utilizados com boa segurança na estimativa da altura das árvores e do volume de madeira (Figura 3).

Os resultados obtidos confirmam o potencial do sensoriamento remoto na estimativa das características da floresta tropical seca, no Semiárido brasileiro. 
$\mathrm{Na}$ análise da reta 1:1 e da linha de tendência, observase que os modelos superestimaram os valores de altura média por parcela e o volume de madeira por hectare, no fragmento de Canindé de São Francisco, e os subestimaram em Porto da Folha. Os valores extrapolados (+ ou -) variaram em torno de $0,07 \mathrm{~m}$ de altura e $4 \mathrm{~m}^{3} \mathrm{ha}^{-1}$. A possibilidade de estimar as variáveis dendrométricas em $100 \%$ da área florestal, no entanto, é a grande vantagem do sensoriamento remoto em comparação ao método convencional de mensuração em parcelas de inventário florestal e equações alométricas. Essa característica torna possível identificar áreas prioritárias para o manejo florestal sustentável do Bioma Caatinga, no Estado de Sergipe, e mostra que o sensoriamento remoto é uma ferramenta importante para órgãos estaduais e federais responsáveis pela fiscalização, pela manutenção e pela conservação desta importante formação florestal, exclusiva do
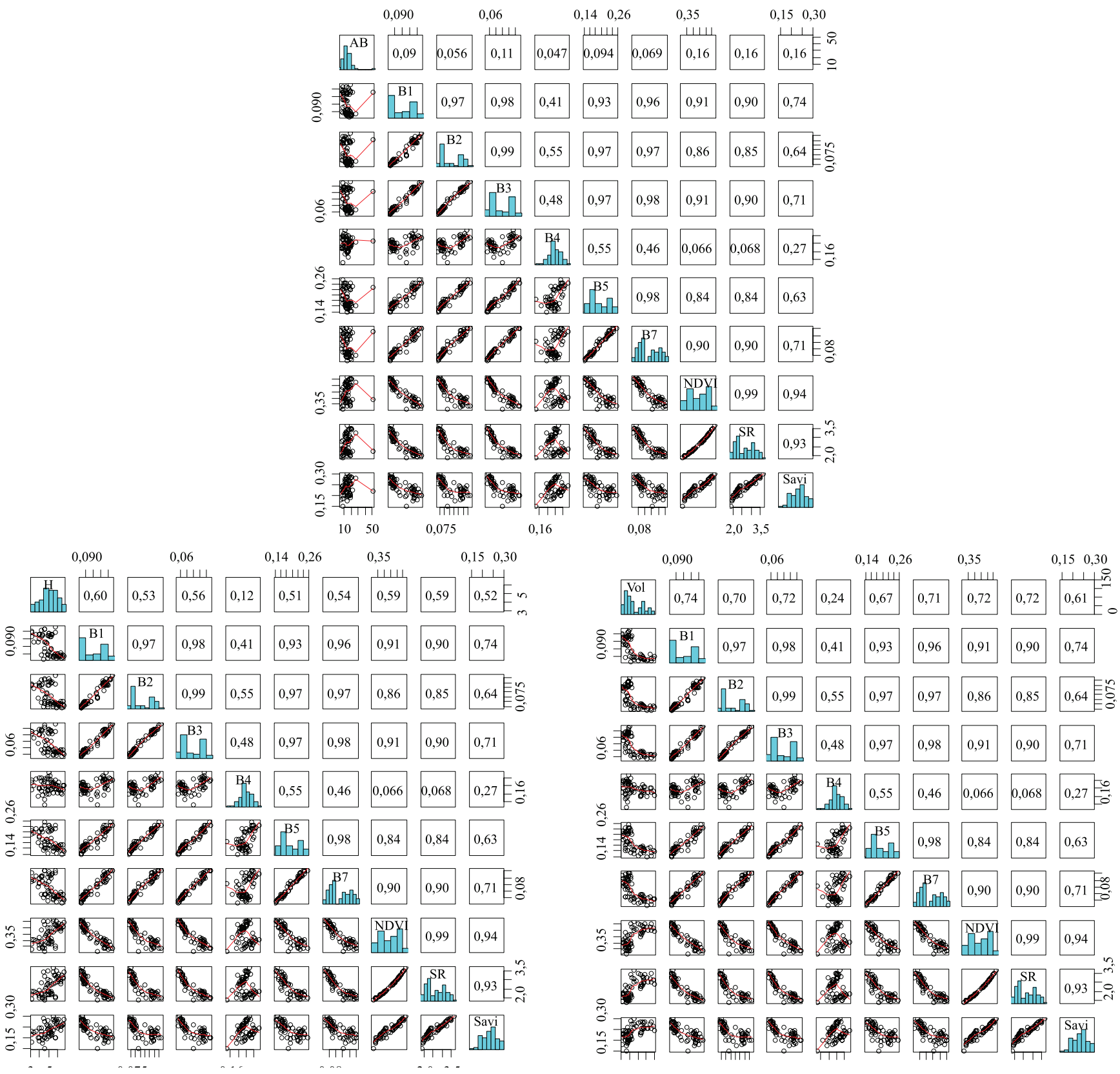

Figura 2. Diagrama de dispersão e coeficientes de correlação entre os valores das variáveis explicativas do satélite Landsat 5 e os de: A, área basal por hectare; B, altura média das árvores; e C, volume de madeira por hectare e variáveis. 
território nacional. Este tipo de trabalho também é importante para a estimativa de biomassa verde acima do solo e, consequentemente, para a estimativa de carbono estocado pela vegetação, que servem como
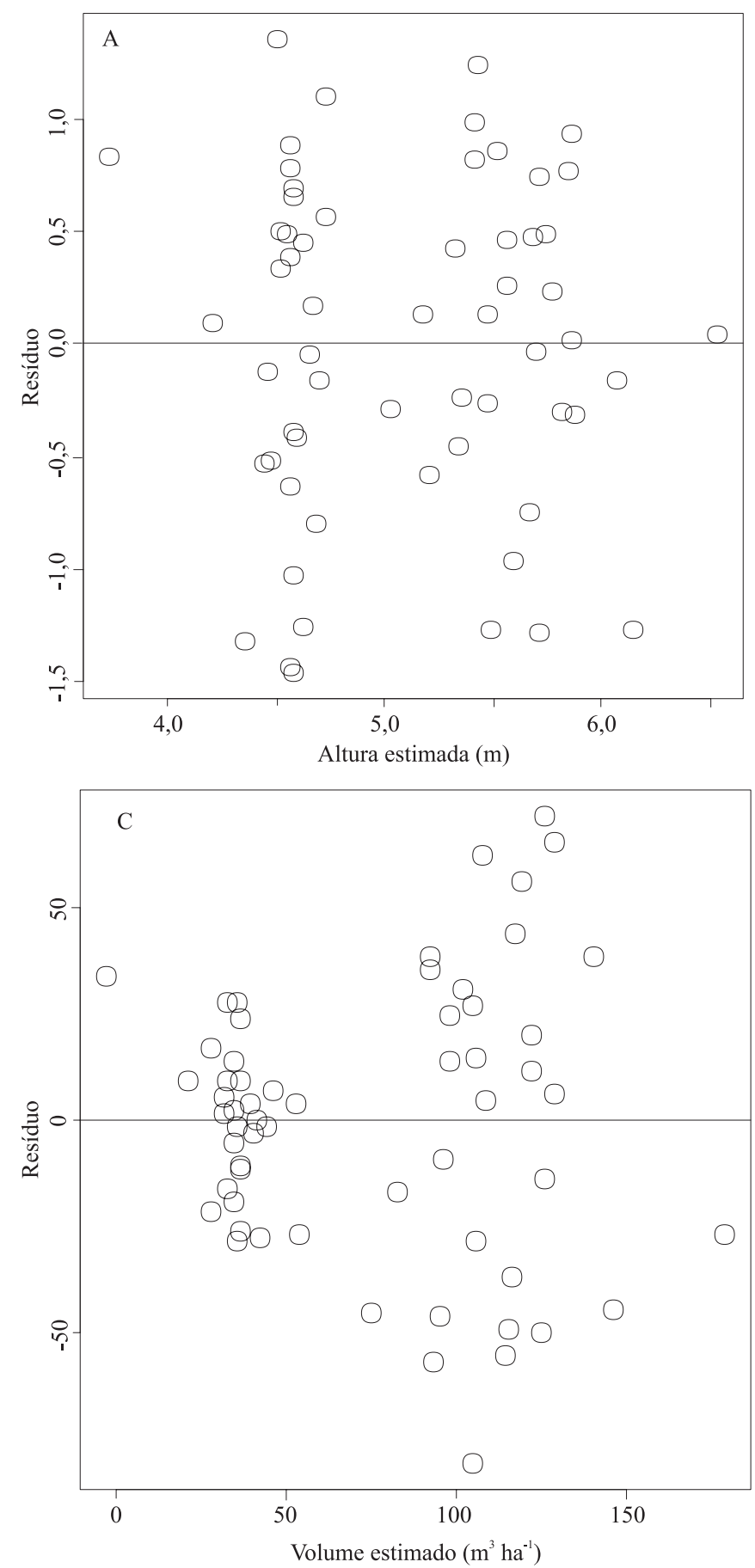

base para a aprovação de projetos de mecanismos de desenvolvimento limpo (Hajek et al., 2011).

O teste de validação cruzada, que considerou $25 \%$ dos dados analisados e 1.000 repetições, confirmou
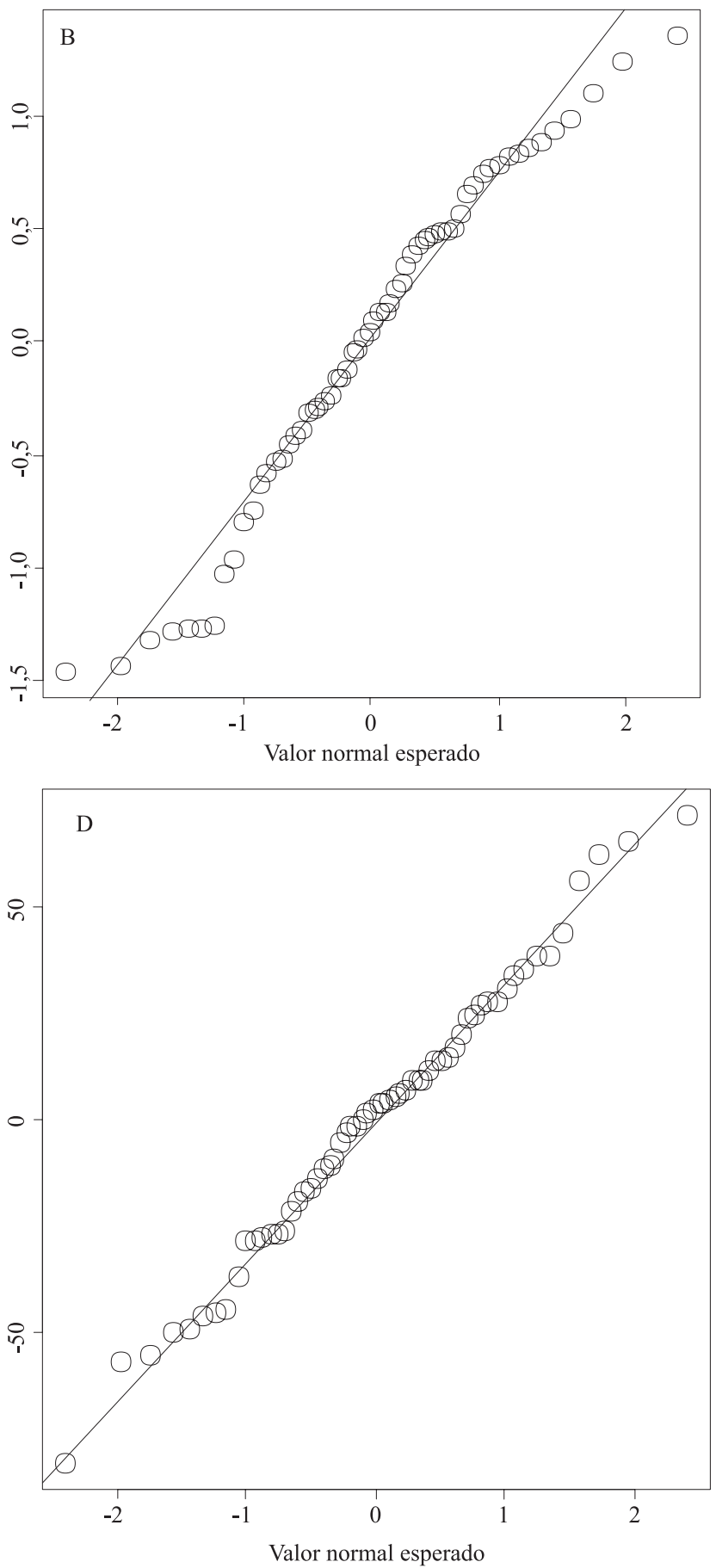

Figura 3. Diagrama de dispersão entre os resíduos e os valores de alturas estimadas (A); gráfico de probabilidade normal dos resíduos das alturas (B); diagrama de dispersão entre os resíduos e os valores de volume estimado (C); e gráfico de probabilidade normal dos resíduos dos volumes (D). 
a validade dos modelos, com valores de $\mathrm{R}^{2}$ e RMSE, respectivamente, de 0,37 e 0,78 , para a altura média das árvores por parcela, e de 0,61 e 34,5 , para o volume de madeira por hectare. O diagrama de dispersão (Figura 4) mostra que os modelos ajustados conseguiram estimar todo o "range" da vegetação, com os menores valores estimados no fragmento de
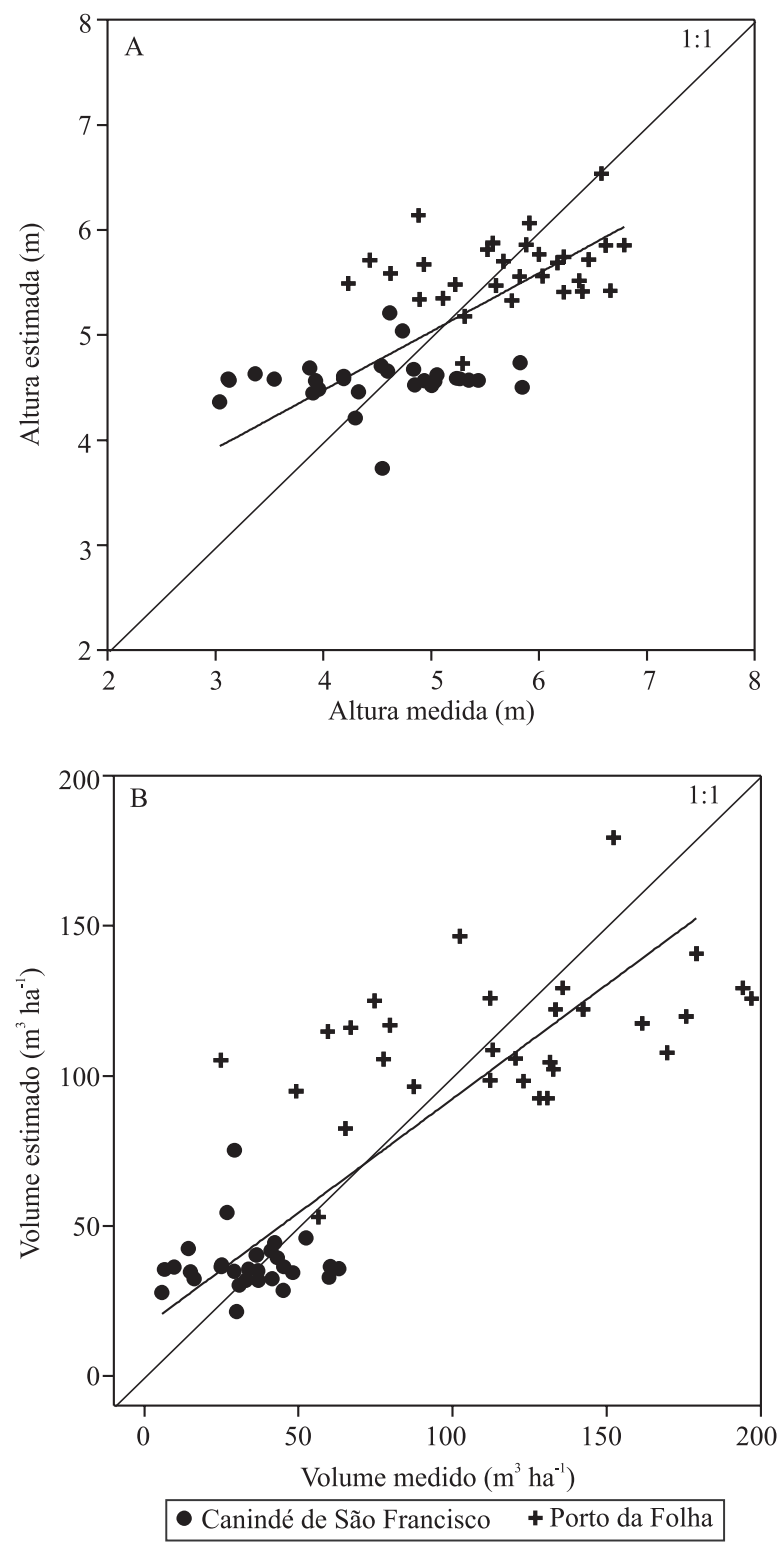

Figura 4. Diagrama de dispersão entre os valores de: A, altura medida vs. estimada; e B, volume medido vs. estimado, na vegetação de Caatinga do Semiárido brasileiro. Ajuste da linha de tendência e da linha 1:1.
Canindé de São Francisco e os maiores no de Porto da Folha. Os modelos, portanto, corroboraram as métricas que caracterizavam ambos os fragmentos (Tabela 1). Outro importante resultado observado foi a não saturação dos dados sensoriais (Huete et al., 1997), ocorrência comum em trabalhos com florestas densas, como as tropicais, que apresentam elevados valores de biomassa verde acima do solo (Dong et al., 2003).

Trabalhos futuros devem considerar, além das variáveis explicativas analisadas no presente trabalho, outros índices derivados do sensor TM do Landsat, bem como de outros sensores orbitais, com melhor resolução espacial e temporal. Além disso, outras técnicas de sensoriamento remoto devem ser testadas, como o "light detection and ranging" (Lidar). Outro ponto importante a ser destacado é o tamanho das parcelas de inventário florestal: recomenda-se que a área inventariada em cada parcela seja de, no mínimo, 0,5 ha.

\section{Conclusões}

1. As variáveis explicativas obtidas do sensor TM do satélite Landsat 5 não explicam a variação em área basal por hectare.

2. A altura das árvores por parcela e o volume de madeira por hectare estão correlacionados às variáveis explicativas utilizadas.

3. A banda vermelha do espectro eletromagnético e os índices NDVI e Savi, derivados do Landsat 5, são as principais variáveis que explicam a variação em altura e volume de madeira por hectare, para a vegetação de Caatinga analisada.

4. Os dados do sensor TM não apresentam saturação, na vegetação de Caatinga analisada.

\section{Referências}

ACCIOLY, L.J. de O.; PACHÊCO, A.; COSTA, T.C. e C. da; LOPES, O.F.; OLIVEIRA, M.A.J. de. Relações empíricas entre a estrutura da vegetação e dados do sensor TM/LANDSAT. Revista Brasileira de Engenharia Agrícola e Ambiental, v.6, p.492-498, 2002. DOI: $10.1590 / \mathrm{S} 1415-43662002000300019$.

ARROYO-MORA， J.P.; SÁNCHEZ-AZOFEIFA， G.A.; KALACSKA, M.E.R.; RIVARD, B. Secondary forest detection in a neotropical dry forest landscape using Landsat 7 ETM+ and IKONOS imagery. Biotropica, v.37, p.497-507, 2005. DOI: 10.1111/j.1744-7429.2005.00068.x. 
BANNARI, A.; MORIN, D.; BONN, F.; HUETE, A.R. A review of vegetation indices. Remote Sensing Reviews, v.13, p.95-120, 1995. DOI: 10.1080/02757259509532298.

DONG, J.; KAUFMANN, R.K.; MYNENI, R.B.; TUCKER, C.J.; KAUPPI, P.E.; LISKID, J.; BUERMANN, W.; ALEXEYEV, V.; HUGHES, M.K. Remote sensing estimates of boreal and temperate forest woody biomass: carbon pools, sources, and links. Remote Sensing of Environment, v.84, p.393-410, 2003. DOI: 10.1016/ S0034-4257(02)00130-X.

DU, H.; CUI, R.; ZHOU, G.; SHI, Y.; XU, X.; FAN, W.; LU, Y. The responses of Moso bamboo (Phyllostachys heterocycla var. pubescens) forest aboveground biomass to Landsat TM spectral reflectance and NDVI. Acta Ecologica Sinica, v.30, p.257-263, 2010. DOI: 10.1016/j.chnaes.2010.08.005.

FEELEY, K.J.; GILlESPIE, T.W.; TERBORGH, J.W. The utility of spectral indices from Landsat ETM+ for measuring the structure and composition of tropical dry forest. Biotropica, v.37, p.508-519, 2005. DOI: 10.1111/j.1744-7429.2005.00069.x.

FERRAZ, R.C.; MELLO, A.A. de; FERREIRA, R.A.; PRATA, A.P. do N. Levantamento fitossociológico em área de Caatinga no Monumento Natural Grota do Angico, Sergipe, Brasil. Revista Caatinga, v.26, p.89-98, 2013.

FLORESTAS do Brasil em resumo: dados de 2007 a 2012. Brasília: Serviço Florestal Brasileiro, 2013. 188p.

FOLEY, J.A.; DEFRIES, R.; ASNER, G.P.; BARFORD, C.; BONAN, G.; CARPENTER, S.R.; CHAPIN, F.S.; COE, M.T.; DAILY, G.C.; GIBBS, H.K.; HELKOWSKI, J.H.; HOLLOWAY, T.; HOWARD, E.A.; KUCHARIK, C.J.; MONFREDA, C.; PATZ, J.A.; PRENTICE, I.C.; RAMANKUTTY, N.; SNYDER, P.K. Global consequences of land use. Science, v.309, p.570-574, 2005. DOI: $10.1126 /$ science. 1111772 .

FORMIGONI, M. de H.; XAVIER, A.C.; LIMA, J.S. de S. Análise temporal da vegetação na região do Nordeste através de dados EVI do MODIS. Ciência Florestal, v.21, p.1-8, 2011.

GAO, X.; HUETE, A.R.; NI, W.G.; MIURA, T. Optical-biophysical relationships of vegetation spectra without background contamination. Remote Sensing of Environment, v.74, p.609-620, 2000. DOI: 10.1016/S0034-4257(00)00150-4.

GASPARRI, N.I.; PARMUCHI, M.G.; BONO, J.; KARSZENBAUM, H.; MONTENEGRO, C.L. Assessing multi-temporal Landsat 7 ETM+ images for estimating above-ground biomass in subtropical dry forests of Argentina. Journal of Arid Environments, v.74, p.1262-1270, 2010. DOI: 10.1016/j.jaridenv.2010.04.007.

GILLESPIE, T.W. Predicting woody-plant species richness in tropical dry forests: a case study from South Florida, USA. Ecological Applications, v.15, p.27-37, 2005. DOI: 10.1890/03-5304.

HAJEK, F.; VENTRESCA, M.J.; SCRIVEN, J.; CASTRP, A. Regime-building for REDD+: evidence from a cluster of local initiatives in south-eastern Peru. Environmental Science and Policy, v.14, p.201-215, 2011. DOI: 10.1016/j. envsci.2010.12.007.
HUETE, A.R. A soil-adjusted vegetation index (SAVI). Remote Sensing of the Environment, v.25, p.295-309, 1988. DOI: 10.1016/0034-4257(88)90106-X.

HUETE, A.R.; LIU, H.Q.; BATCHILY, K.; VAN LEEUWEN, W. A omparison of vegetation indices over a global set of TM images for EOS-MODIS. Remote Sensing of the Environment, v.59, p.440-451, 1997. DOI: 10.1016/S0034-4257(96)00112-5.

HUETE, A.R.; WARRICK, A.R. Assessment of vegetation and soil water regimes in partial canopies with optical remotely sensed data. Remote Sensing of Environment, v.32, p.155-167, 1990. DOI: 10.1016/0034-4257(90)90015-E.

INSTITUTO BRASILEIRO DE GEOGRAFIA E ESTATÍSTICA. Área territorial oficial. 2010. Disponível em: <http://www.ibge. gov.br/home/geociencias/cartografia/default_territ_area.shtm $>$. Acesso em: $20 \mathrm{dez} 2013$.

INSTITUTO BRASILEIRO DO MEIO AMBIENTE E DOS RECURSOS NATURAIS RENOVÁVEIS. Instrução Normativa $\mathrm{n}^{\mathrm{o}} 3$, de 4 de maio de 2001. Ajusta procedimentos relativos ao Manejo Florestal Sustentável da vegetação nativa e suas formações sucessoras na região Nordeste do Brasil. Diário Oficial [da] República Federativa do Brasil, 7 maio 2001.

JUNCÁ, F.A.; FUNCH, L.; ROCHA, W. Biodiversidade e conservação da Chapada Diamantina. Brasília: Ministério do Meio Ambiente, 2005. 411p.

KALACSKA, M.E.R.; SÁNCHEZ-AZOFEIFA, G.A.; CAELLI, T.; RIVARD, B.; BOERLAGE, B. Estimating leaf area index from satellite imagery using Bayesian networks. IEEE Transactions on Geoscience and Remote Sensing, v.43, p.1866-1873, 2005. DOI: 10.1109/TGRS.2005.848412.

LU, D.S. The potential and challenge of remote sensing-based biomass estimation. International Journal of Remote Sensing, v.27, p.1297-1328, 2006. DOI: 10.1080/01431160500486732.

PALACIOS-ORUETA, A.; CHUVIECO, E.; PARRA, A.; CARMONA-MORENO, C. Biomass burning emissions: a review of models using remote sensing data. Environmental Monitoring and Assessment, v.104, p.189-209, 2005. DOI: 10.1007/ s10661-005-1611-y.

POWELL, S.L.; COHEN, W.B.; HEALEY, S.P.; KENEEDT, R.E.; MOISEN, G.G.; PIERCE, K.B.; OHMANN, J.L. Quantification of live aboveground forest biomass dynamics with Landsat time-series and field inventory data: a comparison of empirical modeling approaches. Remote Sensing of Environment, v.114, p.1053-1068, 2010. DOI: 10.1016/j.rse.2009.12.018.

REDE de Manejo Florestal da Caatinga: protocolo de medições de parcelas permanentes. Recife: Associação Plantas do Nordeste, 2005. 21p.

ROSENQVIST, A.; MILNE, A.; LUCAS, R.; IMHOFF, M.; DOBSON, C. A review of remote sensing technology in support of the Kyoto Protocol. Environmental Science and Policy, v.6, p.441-455, 2003. DOI: 10.1016/S1462-9011(03)00070-4.

SÁNCHEZ-AZOFEIFA, G.A.; QUESADA, M.; RODRÍGUEZ, J.P.; NASSAR, J.M.; STONER, K.E.; CASTILLO, A.; GARVIN, T.; ZENT, E.L.; CALVO-ALVARADO, J.C.; KALACSKA, M.E.R.; FAJARDO, L.; GAMON, J.A.; CUEVAS-REYES, P. 
Research priorities for Neotropical dry forests. Biotropica, v.37, p.477-485, 2005. DOI: 10.1046/j.0950-091x.2001.00153.x-i1.

TUCKER, C.J. Red and photographic infrared linear combinations for monitoring vegetation. Remote Sensing of Environment, v.8, p.127-150, 1979. DOI: 10.1016/0034-4257(79)90013-0.

TURNER, D.P.; COHEN, W.B.; KENNEDY, R.E.; FASSNACHT, K.S.; BRIGGS, J.M. Relationships between leaf area index and
Landsat TM spectral vegetation indices across three temperate zone sites. Remote Sensing of Environment, v.70, p.52-68, 1999. DOI: 10.1016/S0034-4257(99)00057-7.

VERMOTE, E.F.; TANRÉ, D.; DEUZÉ, J.L.; HERMAN, M.; MORCRETTE, J.J. Second simulation of the satellite signal in the solar spectrum, 6S: an overview. IEEE Transactions on Geosciences and Remote Sensing, v.35, p.675-86, 1997. DOI: 10.1109/36.581987.

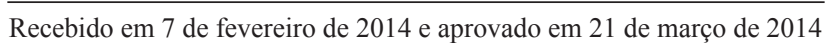

